\title{
Target Object Identification and Location Based on Multi-sensor Fusion
}

\section{Yong Jiang1, *, Hong-Guang Wang1, and Ning $\mathrm{Xi}^{2}$}

${ }^{1}$ Shenyang Institute of Automation, Chinese Academy of Sciences, China

${ }^{2}$ Department of Electrical and Computer Engineering, Michigan State University, USA

(Received 9 October2012; Accepted 25 October 2012; Published on line 1 March 2013)

*Corresponding author: jiangyong@sia.cn

DOI: $\underline{10.5875 / a u s m t . v 3 i 1.171}$

\begin{abstract}
For an unknown environment, how to make a mobile robot identify a target object and locate it autonomously is a very challenging endeavor. In this paper, a novel multi-sensor fusion method based on a camera and a laser range finder (LRF) for mobile manipulations is proposed. Although a camera can acquire large quantities of information, it does not directly get the 3D data of the environment. Moreover, the camera image processing is complex and easily influenced from change in ambient light. In view of the ability of the LRF to directly get the 3D coordinates of the environment and its stability against outside influence, and the superiority of the camera to acquire rich color information, the combination of the two sensors by making use of their advantages is employed to obtain more accurate measurement as well as to simplify information processing. A homogeneous transformation model of the system was built to overlay the camera image with the measurement point cloud of the pitching LRF and to reconstruct the 3D image that includes pixel depth information. Then, based on the combination of the color features from the camera image and the shape features from the LRF measurement data, the autonomous identification and location of target object can be achieved. In order to extract the shape features of the object, a two-step method is introduced, and a sliced point cloud algorithm is proposed for the preliminary classification of the measurement data of the LRF. The effectiveness of the proposed method is validated by experimental testing and analysis carried out on the mobile manipulator platform. The experimental results show that by this method, the robot can not only identify a target object autonomously, but also determine whether it can be operated, and acquire a proper grasping location.
\end{abstract}

Keywords: multi-sensor fusion; mobile manipulations; object identification and location; camera and laser range finder

\section{Introduction}

Mobile manipulators exhibit numerous advantages over traditional fixed-base manipulators. Since they are mounted on a mobile base, the manipulator can significantly increase the workspace of the manipulation, and become more maneuverable and transportable. Due to this, mobile manipulators have applications in many areas, such as in assembly tasks, aerospace, service environments, and nuclear power stations [1-4].

When performing a dangerous task, like disposing of explosives, many actions need to be done autonomously by the mobile manipulator with no humans involved. There are several reasons for this. First, the working environments can be hazardous, unstructured, or unknown in advance. Additionally, dynamic change often happens to the environment. A walking person or a moving object often makes the environment become more complex. The operating target in the workspace may be not sure or be confused with the complicated scene. Therefore, the robot has to decide where to go, whether it is the target object or not, how to navigate to reach for it, how and where to grasp it, how to hold it, and so on. The capacity for autonomous target object identification and location is a 
very important function to improve the performance of the mobile manipulations. This is also a very challenging question for such a system.

Many attempts have been made to achieve autonomy. Various sensors are usually equipped on the mobile manipulator, particularly a camera and a laser range finder (LRF). Takeshi Nishida et al. developed a sensor system for an outdoor service robot, OSR-02 [5]. This sensor system consists of a LRF and two cameras. The camera image processing method is employed for detection and tracking of the target object. The PSF method is presented to extract the data set of the target object from the LRF measurement data set for the analysis of the object. S. Datta et al. developed a mobile manipulator that can navigate autonomously and transport jobs and tools in a manufacturing environment [6]. Here, a CMOS camera mounted on the manipulator identifies jobs for pick-and-place operations. A variation of correlation based adaptive predictive search method is used for job identification. Andrea Gasparri et al. developed a multi-robot system composed of a 5-DOF manipulator and a mobile base [7]. The system is equipped with some exteroceptive sensors like sonar and LRF. A color camera is mounted on the end-effector of the manipulator to locate objects to operate with. An ultrasound range finder, near the camera, is used to get distance measures. Garcia-Cerezo et al. presented a mobile manipulator ALACRANE assistant for exploration

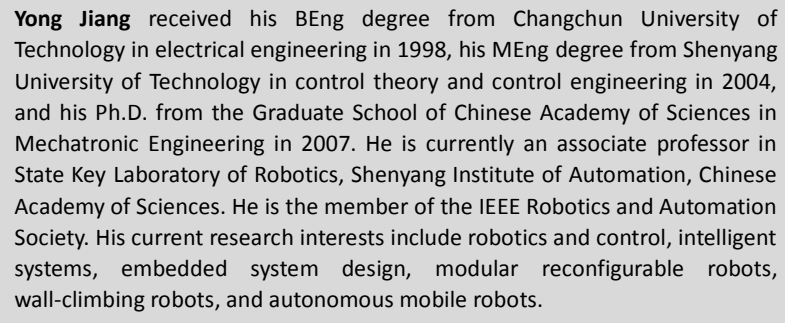
Technology in electrical engineering in 1998, his MEng degree from Shenyang University of Technology in control theory and control engineering in 2004, and his Ph.D. from the Graduate School of Chinese Academy of Sciences in Mechatronic Engineering in 2007. He is currently an associate professor in State Key Laboratory of Robotics, Shenyang Institute of Automation, Chinese Academy of Sciences. He is the member of the IEEE Robotics and Automation Society. His current research interests include robotics and control, intelligent systems, embedded system design, modular reconfigurable robots, wall-climbing robots, and autonomous mobile robots.

Hong-Guang Wang received his BEng degree from Zhengzhou Institute of Technology in 1986, his MEng degree from Northeastern University in Mechanics in 1993, and his Ph.D. from the Graduate School of Chinese Academy of Sciences in Mechatronic Engineering in 2008. He is currently a professor in State Key Laboratory of Robotics, Shenyang Institute of Automation, Chinese Academy of Sciences. He is the member of the IEEE Robotics and Automation Society. His current research interests include analysis and synthesis of robot mechanism, mechanics of serial and parallel manipulators, modular reconfigurable robots, and autonomous mobile robots.

Ning $\mathbf{X i}$ received his DSC degree in Systems Science and Mathematics from Washington University in St. Louis, USA, in December, 1993. He received his M.S. degree in computer science from Northeastern University, Boston, USA, and his B.S. degree in electrical engineering from Beijing University of Aeronautics and Astronautics, China. Currently, he is a professor of electrical and computer engineering in the Department of Electrical and Computer Engineering at Michigan State University, USA. He is the fellow of IEEE, a member of ASME, a member of SPIE, the Editor of International Journal of Micro/Nano Mechatronics, and a member of Editorial Board of International Journal of Advanced Robotics. His current research interests include robotics, manufacturing automation, micro/nano systems, intelligent control and systems, and biomedical applications. and rescue missions [8]. The system is equipped with CCD and thermal cameras and a LRF. The CCD and thermal cameras are used together to determine the possible targets. The LRF offers the main functions in navigation mode. Manabu Yamashiro et al. describe a mobile manipulator for home service [9]. Its sensor system consists of radio frequency identification devices (RFID), stereo camera and LRF. To realize the recognition of various objects as well as to get more information, the sensor fusion method of RFID and stereo camera was proposed. By referring the model of the chair from a database related to the ID, the detection of handling point of the chair is discussed based on RFID and LRF [9]. Hai Nguyen et al. presented a behavior selection system for human-robot interaction of the mobile manipulator $\mathrm{El}-\mathrm{E}$, which has a laser-pointer interface that detects when a user illuminates a location with an off-the-shelf green laser pointer in the environment and estimates its 3D location by its stereo pan/tilt camera [10]. Aiming at the same platform, Travis Deyle et al. proposed a mode of perception that produces images of the spatial distribution of received signal strength indication (RSSI) for each of the tagged objects [11]. Additionally, a framework for estimating a tagged object's 3D location using fused ID-specific features derived from an RSSI image, a camera image, and a LRF was presented. Ulrich Klank et al. proposed a 3D perception system architecture that can robustly fit CAD models in cluttered table setting scenes for the purpose of grasping with a mobile manipulator [12]. This approach uses a powerful combination of two different camera technologies, Time-of-flight and RGB, to robustly segment the scene and extract object clusters.

For an unknown environment, how to make the mobile manipulator identify a target object and locate it autonomously is a very big challenge. Although the camera can acquire rich information such as color, shape, etc, it does not directly get the 3D data of the environment. Moreover, the camera image processing is complex and easily influenced from the change in ambient light, meaning the target identification and location done only by the camera may be not robust. Due to some auxiliary constraints in the environment, the target object identification and location can be easily realized for special applications, such as the RFID-based method for home service and the laser-pointer interface for human-robot interaction. However, a similar method based on the auxiliary constraints may be not feasible for more general applications. In view of the ability of the LRF to directly measure the 3D coordinates of the environment and its stability against outside influence, the combination of a camera and LRF, making use of their 
advantages, is an available way to obtain more precise measurement and to simplify information processing.

In this paper, a novel information fusion method using a camera and LRF for the mobile manipulator is proposed. A homogeneous transformation model of the system was built to overlay the camera image with the measurement point cloud of the LRF pitching scan to reconstruct the $3 \mathrm{D}$ image including depth information. Then, based on the combination of the color features from the camera image and the shape features from the LRF measurement data, the identification and location of the target object is achieved. In order to extract the shape features of the object, a two-step method is introduced, and a sliced point cloud (SPC) algorithm is proposed for the preliminary classification of the measurement data of the LRF.

\section{Multi-sensor Fusion Method}

\section{Multi-sensor fusion method}

The flowchart of camera and LRF fusion for target object identification and location is shown in Figure 1. The procedure is divided into three stages: information preprocessing, target object identification, and target object location.
1) Information Preprocessing Stage: First, the raw single-frame image from the camera and the raw measurement point cloud from the pitching LRF are obtained. Then, based on the calibration of the two sensors, the point cloud is overlaid onto the raw camera image by using the homogeneous transformation Equations (4), (6), (7) and (8). The reconstructed 3D image including depth information is generated.

2) Target Object Identification Stage: For the objects in the $3 \mathrm{D}$ image, both the color features, such as pixel RGB value and color histogram, and the shape features, such as height and width, are extracted. In order to extract the shape features from the measurement point cloud, a two-step method, which is presented below, is proposed. Then, based on the combination of the color features and the shape features, the target object identification is executed by referring to the feature database. If the target object is found, the next stage is performed; otherwise, start over.

3) Target Object Location Stage: Based on the shape features of the target object and the end-effector model, it is determined whether the manipulator can operate the target.

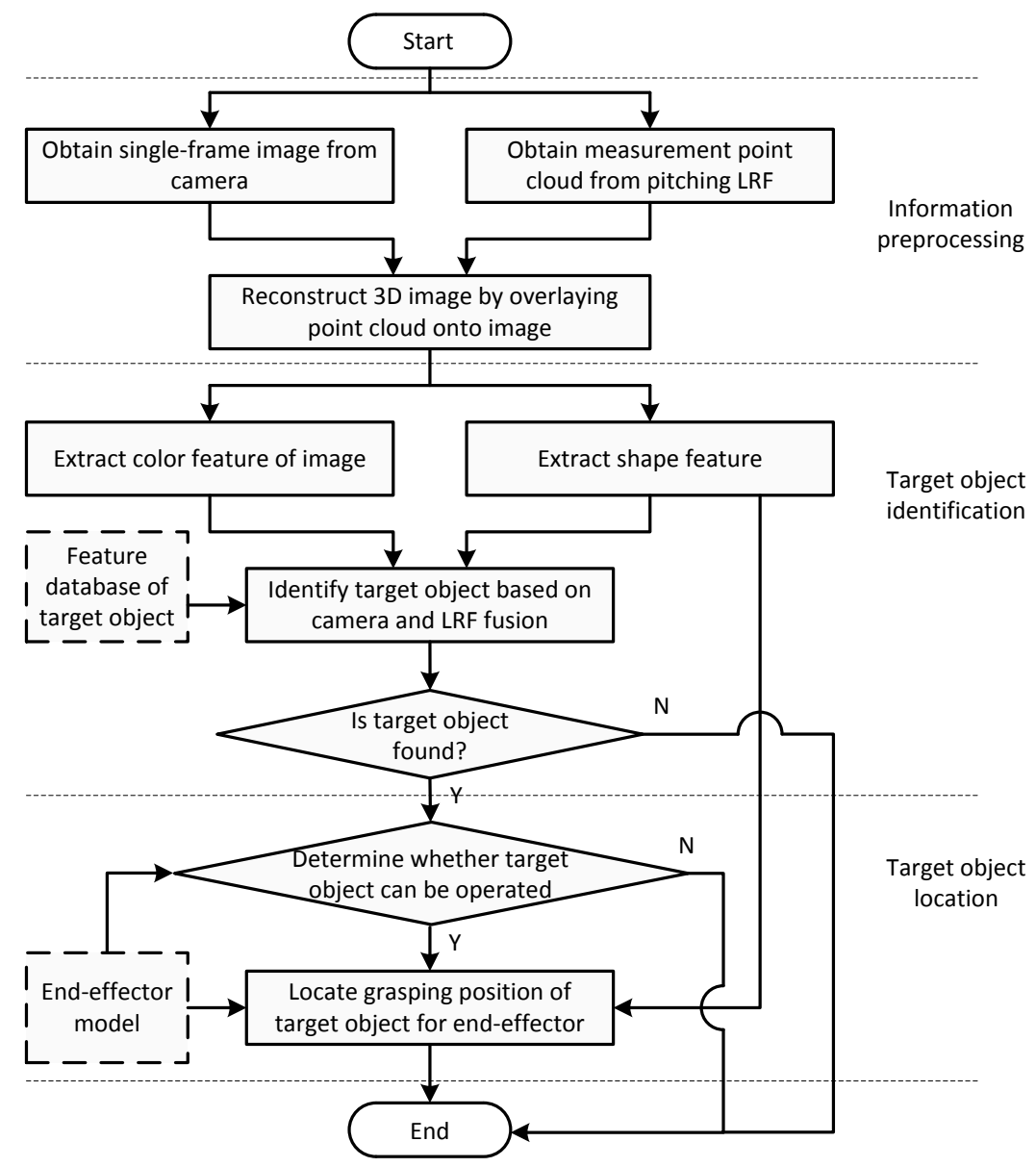

Figure 1. Flowchart. 




Figure 2. Target object location.

In this research, a 2-finger parallel gripper is employed as the end-effector, so the relative size between the maximum stroke of the gripper and the minimum width of the shape features is used for the judgment, as shown in Figure 2.

\section{Shape feature extraction}

To analyze the range data for shape feature extraction and target location, it is necessary to extract the data set of the target object from the measurement data set of the LRF. In this research, the process of shape feature extraction is divided into two steps.

\section{Step 1: Preliminary classification of the measurement data of the LRF}

Let $D$ represent the set of the measurement point cloud overlaid onto the camera image, and ${ }^{p x} p_{i j}$ represent an element of the set $D$. If ${ }^{1 p} p_{i j}$ is the LRF measurement point corresponding to the point ${ }^{p x} p_{i j}$, the pixel depth information implied and the point ${ }^{p x} p_{i j}$ can be found by Equation (1) and

$$
\left[\begin{array}{c}
{ }^{r} p_{i j} \\
1
\end{array}\right]={ }^{r} T\left[\begin{array}{c}
{ }^{\prime} p_{i j} \\
1
\end{array}\right]
$$

where ${ }^{r} p_{i j} \in \Sigma_{r}$ and ${ }^{\prime} p_{i j} \in \Sigma_{l}$. According to the definitions of the coordinate frames in Figure 3, the value ${ }^{r} x_{i j}$ characterizes the pixel depth of the point ${ }^{p x} p_{i j}$. Then, a simple depth-based classifier is employed to classify the measurement point cloud set $D$ into several SPCs. The classifier is defined as

$$
{ }^{D} S_{k}=\left\{{ }^{p x} p_{i j} \mid(k-1) \lambda<{ }^{r} x_{i j} \leq k \lambda\right\},(k=1, \cdots, n),
$$

where $\lambda$ is a presetting slice thickness constant. By using Equation (2), the points in the set $D$ are extracted and divided into $n$ subsets. Each subset represents a SPC. Given the $k^{\text {th }} \mathrm{SPC}{ }^{D} S_{k}$, a corresponding $I \times J$ matrix ${ }^{D} M_{k}$ is defined, in which the element $m_{i j}$ is

$$
m_{i j}=\left\{\begin{array}{ll}
0 & { }^{p x} p_{i j} \notin{ }^{D} S_{k} \\
1 & { }^{p x} p_{i j} \in{ }^{D} S_{k}
\end{array}(i=1, \cdots, I ; j=1, \cdots, J) .\right.
$$

Obviously, each element with a value of 1 in the matrix ${ }^{D} M_{k}$ corresponds to a point in the SPC ${ }^{D} S_{k}$.

\section{Step 2: Segmentation and edge detection}

The segmentation and edge detection of the measurement point cloud in the 3D depth-of-field image are critical for the shape feature extraction. After the preliminary classification, the segmentation and edge detection are performed for each SPC ${ }^{D} S_{k}$ by using the triangular facet normal vector clustering algorithm [18].

\section{Multi-sensor System for Mobile Manipulations}

A mobile manipulator system is usually composed of a mobile base, a manipulator, and a sensor unit including camera and LRF. There are no commercial 3D LRFs available that are suitable for applications of mobile manipulations. A common solution to make $3 \mathrm{D}$ scanning feasible is to use a standard 2D LRF and a mechanical actuator to reach the third dimension, such as pitching scan, rolling scan and yawing scan [13]. In this research, the pitching scan is adopted by using a Sick LMS111 2D LRF and a Schunk PW-70 pan tilt actuator. The configuration and the coordinate frames of the system are shown in Figure 3. Table 1 shows the definition of each coordinate frame.

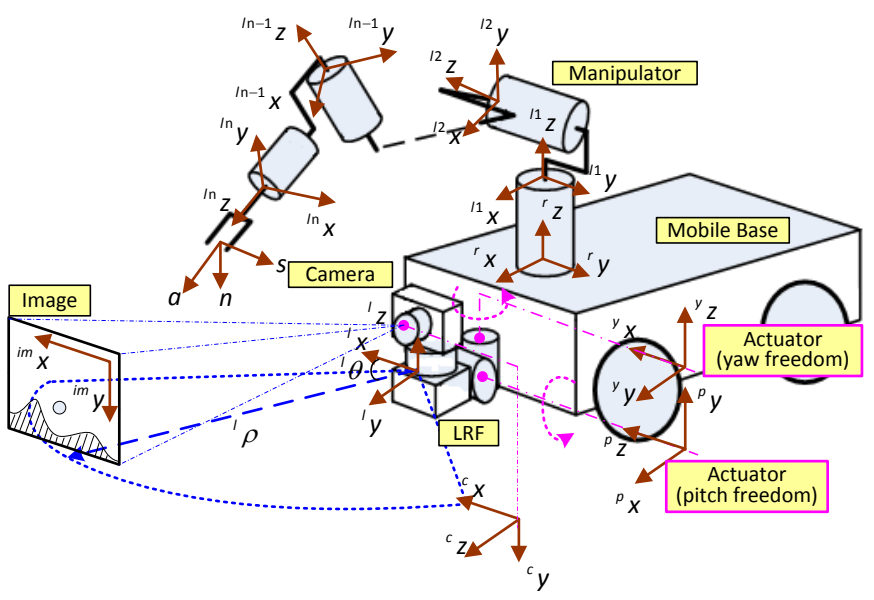

Figure 3. Configuration and coordinate frames.

Table 1. Definitions of Coordinate Frames.

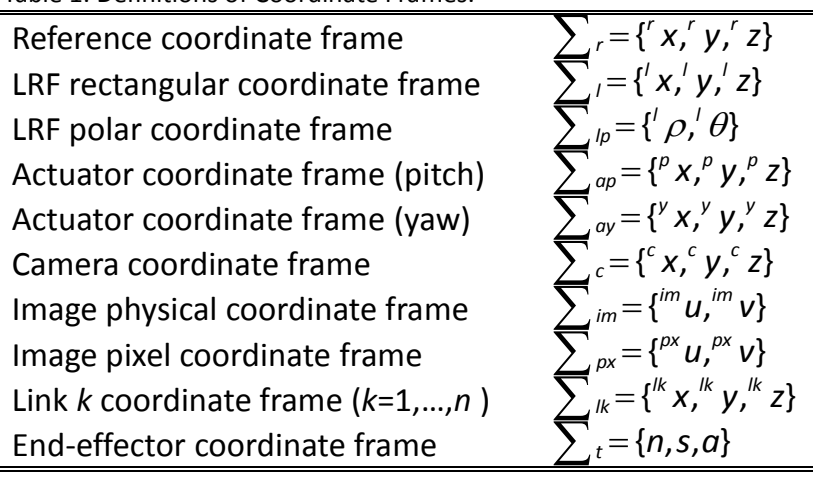


System modeling

The kinematic model of the manipulator is defined as

$$
{ }_{t}^{r} T={ }_{11}^{r} T_{12}^{11} T \ldots{ }_{1 \mathrm{n}}^{\mathrm{ln}-1} T_{t}^{\mathrm{ln}} T
$$

Let ${ }^{1 p} p_{i j}=\left({ }^{\prime} \rho_{i j},{ }^{\prime} \theta_{i j}\right)^{T} \in \Sigma_{l p}$ be the measurement points of the LRF. The mapping from ${ }^{1 p} p_{i j}$ to ${ }^{\prime} p_{i j}=\left({ }^{\prime} x_{i j},{ }^{\prime} y_{i j},{ }^{\prime} z_{i j}\right)^{T} \in \Sigma_{l}$ is represented by

$$
\left\{\begin{array}{l}
{ }^{\prime} x_{i j}={ }^{\prime} \rho_{i j} \cos ^{\prime} \theta_{i j} \\
{ }^{\prime} y_{i j}={ }^{\prime} \rho_{i j} \sin ^{\prime} \theta_{i j} . \\
{ }^{\prime} z_{i j}=0
\end{array}\right.
$$

The mapping from ' $p_{i j}$ to ${ }^{t} p_{i j} \in \Sigma_{t}$ and ${ }^{c} p_{i j} \in \Sigma_{c}$ is given by

$$
\begin{aligned}
& {\left[\begin{array}{c}
{ }^{t} p_{i j} \\
1
\end{array}\right]={ }_{i}^{t} T\left[\begin{array}{c}
{ }^{\prime} p_{i j} \\
1
\end{array}\right],} \\
& {\left[\begin{array}{c}
{ }^{c} p_{i j} \\
1
\end{array}\right]={ }^{c} T\left[\begin{array}{c}
{ }^{\prime} p_{i j} \\
1
\end{array}\right] .}
\end{aligned}
$$

The mapping from ${ }^{c} p_{i j}$ to ${ }^{i m} p_{i j} \in \Sigma_{i m}$ is represented as

$$
{ }^{c} z_{i j}\left[\begin{array}{c}
{ }^{i m} p_{i j} \\
1
\end{array}\right]=\left[\begin{array}{cccc}
f_{c} & 0 & 0 & 0 \\
0 & f_{c} & 0 & 0 \\
0 & 0 & 1 & 0
\end{array}\right]\left[\begin{array}{l}
{ }^{c} p_{i j} \\
1
\end{array}\right],
$$

where $f_{c}$ is the focal length of the camera. The mapping from ${ }^{i m} p_{i j}$ to ${ }^{p x} p_{i j} \in \Sigma_{p x}$ is represented as

$$
\left[\begin{array}{c}
{ }^{p x} p_{i j} \\
1
\end{array}\right]=\left[\begin{array}{ccc}
s_{x} & 0 & u_{o} \\
0 & s_{y} & v_{o} \\
0 & 0 & 1
\end{array}\right]\left[\begin{array}{c}
{ }^{i m} p_{i j} \\
1
\end{array}\right],
$$

where $s_{x}, s_{y}, u_{o}$ and $v_{o}$ are the interior parameters of the camera and related with its internal structure.

\section{Calibration}

Because of the unknown offset between the pitching axis and the LRF origin, as well as the initial pose error which is difficult to measure directly, the calibration between the pan/tilt actuator and the LRF need to be carried out to increase the measurement accuracy of the system. Here the calibration algorithm introduced in [14] is referred. Its principle is shown in Figure 4.

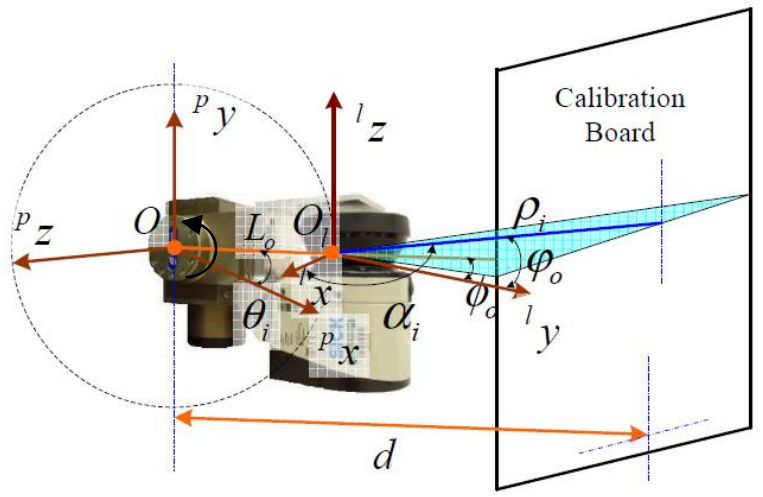

Figure 4. Calibration principle.

Equation (9) is a target function, where $L_{0}$ represents the length between the pitching axis $O$ and the real origin $O_{l}$ of the LRF; $\varphi_{o}$ the angle between the ' $y$ axis of the LRF rectangular coordinate frame $\Sigma_{l}=\left\{{ }^{\prime} x,{ }^{\prime} y,{ }^{\prime} z\right\}$ and the line $O O_{l} ; \phi_{0}$ the initial pose error of the pitching angle; $\rho_{i}$ the ranging data of the LRF; $\theta_{i}$ the nominal pitching angle; $\alpha_{i}$ the scanning angle of the LRF; $\beta$ the angle between the ' $x$ axis of $\Sigma_{l}$ and the surface of the calibration board; and $d$ the distance between the axis $O$ and the surface of the calibration board. The calibration parameters $L_{0}, \varphi_{o}$ and $\phi_{0}$ can be obtained by minimizing Equation (9) based on the Levenberg-Marquardt algorithm in [15].

$$
\begin{aligned}
& f\left(L_{o}, \varphi_{o}, \phi_{o}, d, \beta\right) \\
& =L_{o} \cos \beta \cos \left(\theta_{i}+\phi_{o}-\varphi_{o}\right) \\
& +\rho_{i} \sin \left(\alpha_{i}-\beta\right) \cos \left(\theta_{i}+\phi_{o}\right)-d .
\end{aligned}
$$

The Matlab Calibration Toolbox is used to estimate the intrinsic parameters of the camera. Since the measurements of the camera and the LRF cannot be consistent with each other directly, to realize the fusion of the two sensors, it is absolutely necessary to get the precise model of the homogeneous coordinate transform matrix between the coordinate frames associated to them. In this research, the extrinsic calibration method described in [16] is used.

\section{Experimental Testing and Analysis}

To validate the proposed method and algorithm, some experiments were carried out on the mobile manipulator platform.

\section{Experimental platform}

The mobile manipulator platform is composed of a four-wheel mobile base, a Schunk modular manipulator, and a sensor unit, as shown in Figure 5 . The sensor unit consists of a LRF, a CCD camera and a pan/tilt actuator. 
The SICK LMS111 is an outdoor electro-optical laser measurement system that electro-sensitively scans the perimeter of its surroundings in a plane with the aid of a laser beam. The infrared laser beam is radially irradiated from the center part of the LRF. Its measurement distance is maximum $20 \mathrm{~m}$, the scanning range up to $270^{\circ}$, and the angular resolution $0.25^{\circ}$ or $0.5^{\circ}$. The CCD color camera captures images in 24-bit RGB color at rates up to $30 \mathrm{fps}$. Its resolution is $640 \times 480$ pixels. The Schunk PW-70 servo-electric pan/tilt actuator is a rotary module with two rotating axes for precise positioning. The rotation range of its pitch axis is $\pm 120^{\circ}$, and the yaw axis is $360^{\circ}$.

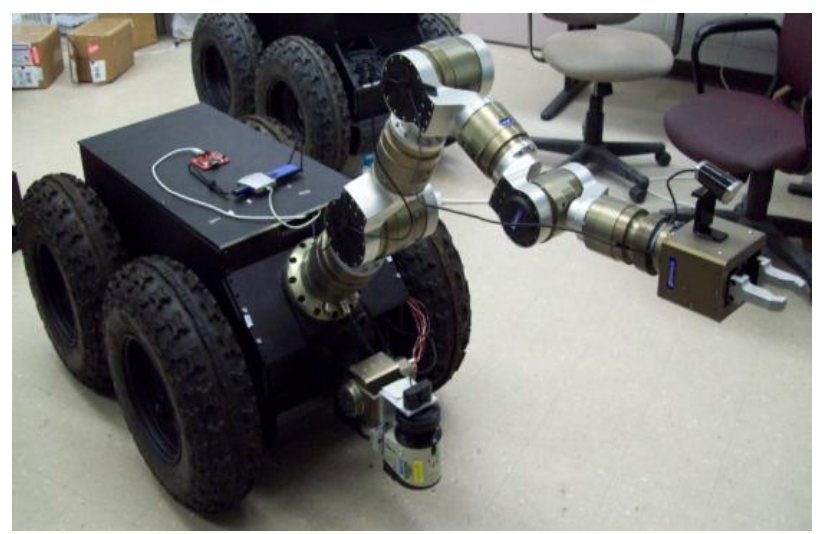

Figure 5. Experimental platform.

The calibration results of the sensor unit on the mobile manipulator are as follows:

- The parameters between the LRF and the pan tilt actuator are: $L_{o}=147.8 \mathrm{~mm}, \varphi_{o}=2.4^{\circ}, \phi_{o}=3.1^{\circ}$.

- The intrinsic parameters of the camera are given by

$$
\left\{\begin{array}{l}
{ }^{p x} u_{i j}=830.22 \frac{{ }^{c} X_{i j}}{{ }^{c} z_{i j}}+284.96 \\
{ }^{p x} v_{i j}=835.24 \frac{{ }^{c} y_{i j}}{{ }^{c} z_{i j}}+215.98
\end{array} .\right.
$$

- The homogeneous coordinate transform matrix between the camera and the LRF is given by

$$
{ }_{c}{ }^{c} T=\left[\begin{array}{cccc}
999.2 & -38.1 & 10.8 & -27.1 \\
38.0 & 999.2 & 14.8 & -96.6 \\
-11.3 & -14.4 & 999.8 & 21.9 \\
0 & 0 & 0 & 1
\end{array}\right]
$$

\section{Experimental testing and analysis}

Figure 6 shows the testing results of the 3D color image reconstruction. The depth-of-field information in each image contains a mass of ranging data by pitching

www.ausmt.org

Copyright (C) 2013 International Journal of Automation and Smart Technology scan of the LRF. The objects in these images, such as paint can, calibration board, and tool box, indicate not only the color features, but also the depth information relative to the mobile manipulator. The nearer the object is to the robot, the redder the point color is. The farther the object is from the robot, the bluer the point color is. Therefore, different from the ordinary 2D image, these are reconstructed 3D color images including the depth-of-field information.
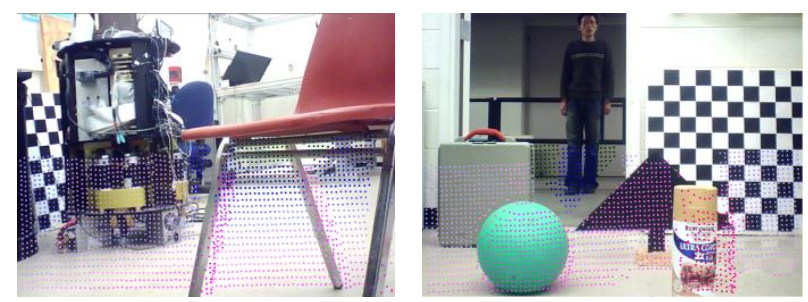

Figure 6. 3D reconstructed color images.

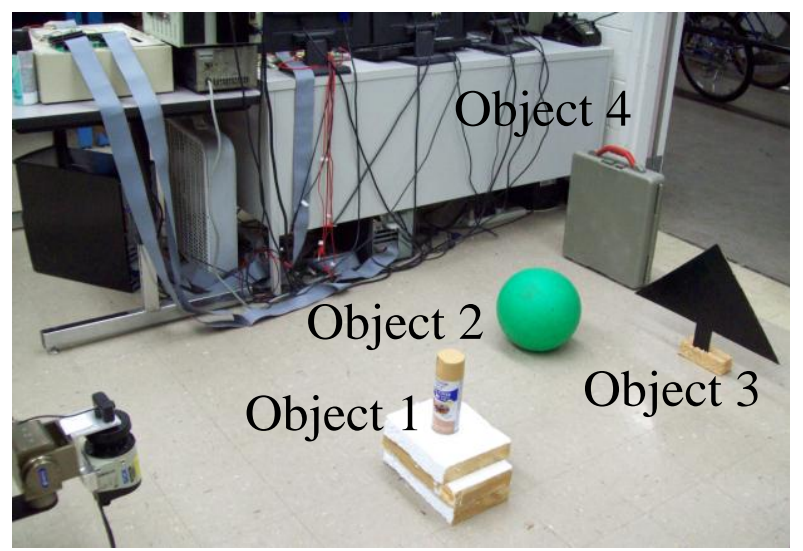

Figure 7. Experimental objects.
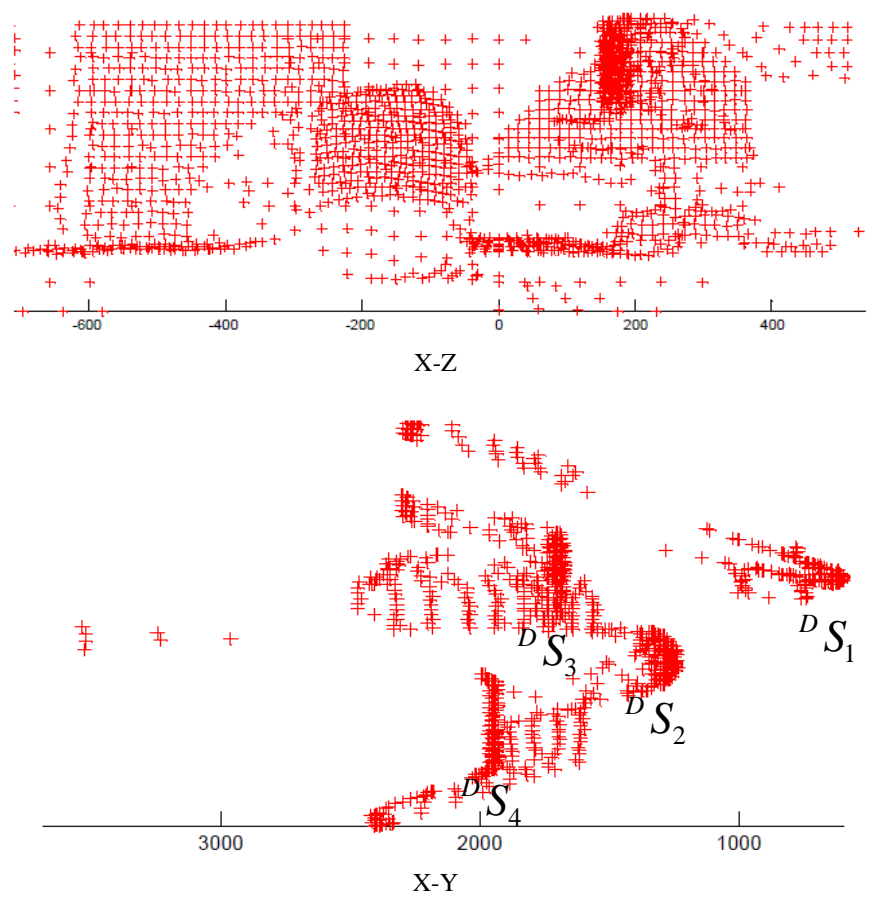

Figure 8. Measurement point cloud. 


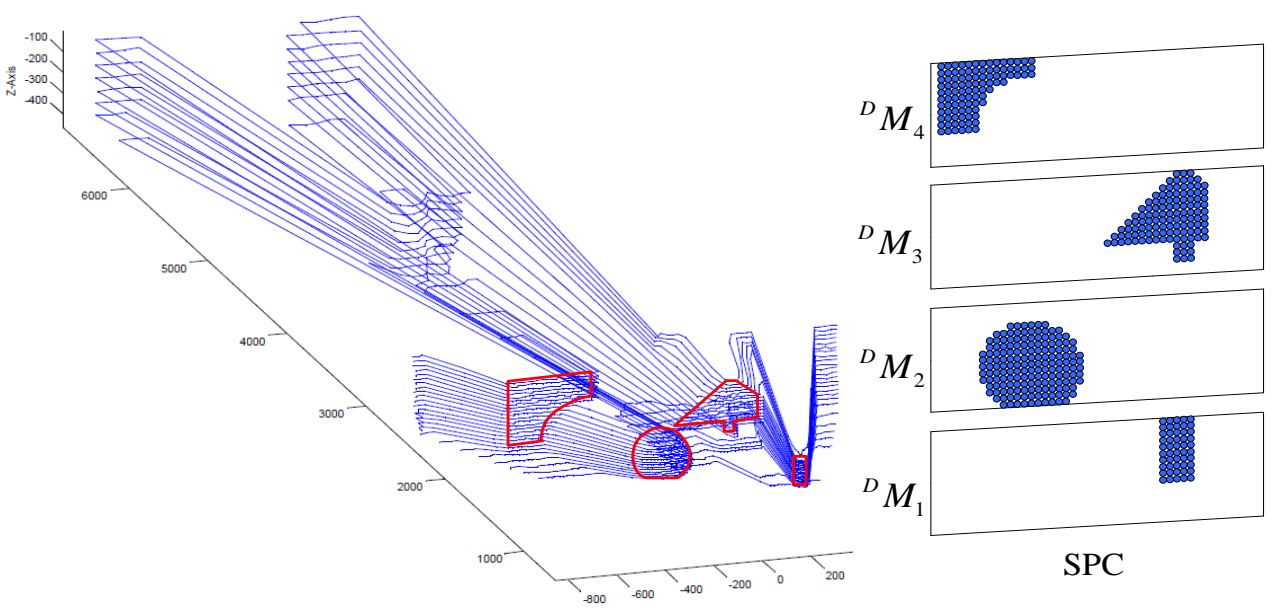

Figure 9. SPCs and matrixes.

Figures 7, 8 and 9 show the experimental results of the SPC method. It can be seen that the point cloud in the $3 D$ image is divided into four SPCS, and the corresponding matrices are ${ }^{D} M_{1},{ }^{D} M_{2},{ }^{D} M_{3}$ and ${ }^{D} \mathrm{M}_{4}$.

Figure 10 shows the testing results of segmentation and edge detection of the 3D depth-of-field image.

Figure 11 presents the user operation interface of the mobile manipulator platform. Figure 12 shows the experiment results of target object location and grasping. It can be seen that by the proposed method, the mobile manipulator cannot only determine whether the target object can be operated, but also acquire a proper grasping location based on the shape features and the gripper model.

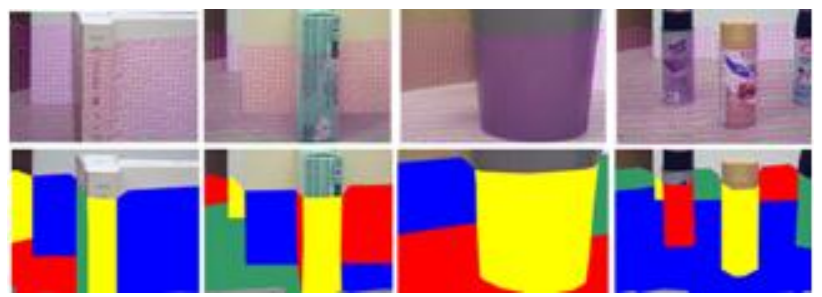

Figure 10. Segmentation and edge detection.

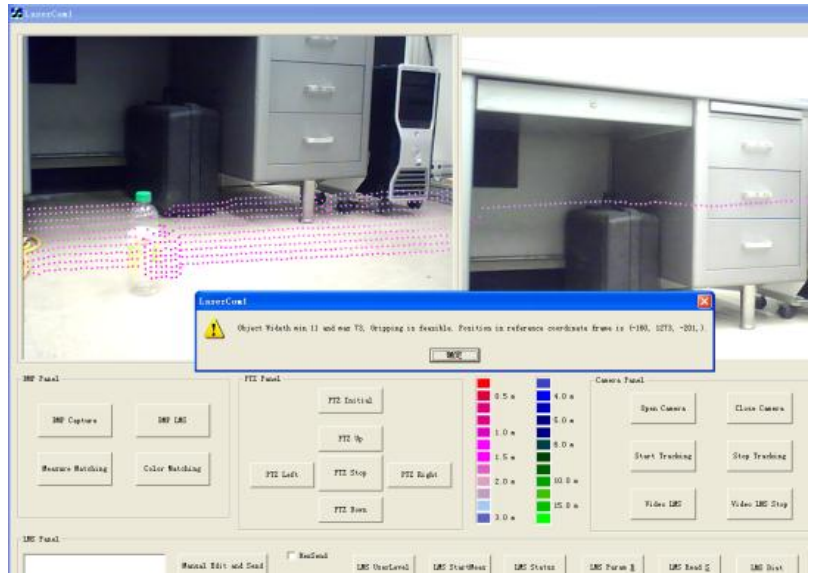

Figure 11. User operation interface (part).

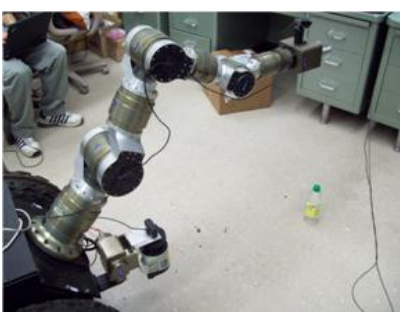

(a)

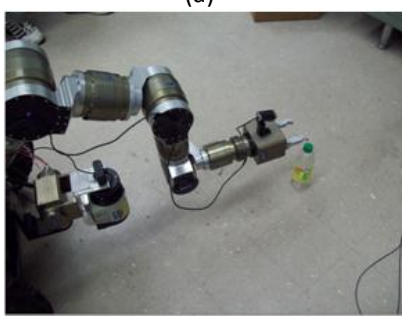

(c)

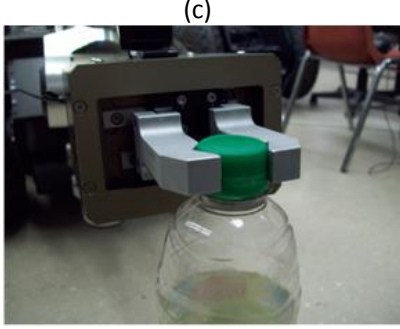

(e)

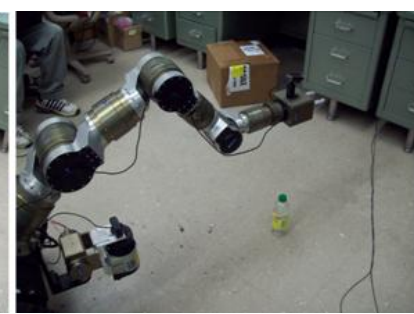

(b)



(d)

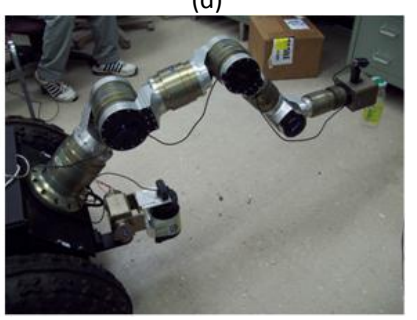

(f)
Figure 12. Experiment results of target location.

\section{Conclusion}

A novel multi-sensor fusion method is proposed in this paper aiming at target object identification and location for mobile manipulators. System modeling and calibration were constructed to overlay the camera image with the measurement point cloud of the LRF pitching scan and reconstruct the 3D image including the pixel depth information,. Then, based on the combination of the color features from the camera image and the shape features from the LRF measurement data, the identification and location of the target object are achieved. In order to extract the shape features of the 
object, a two-step method is introduced, and a SPC algorithm is proposed for the preliminary classification of the measurement data of the LRF. The experiments carried out on the mobile manipulator platform validate the effectiveness of the proposed method. The experimental results show that using this method, a robot can identify and locate a target object autonomously in an unknown environment, and the method is more robust accounting for outside influence. Further developments of this work will be devoted to study path planning and dynamic obstacle avoidance for the mobile manipulator based on the multi-sensor information fusion.

\section{Acknowledgements}

This work was supported in part by the NSFC (Natural Science Foundation of China) 60905048 and was developed in the Department of Electrical and Computer Engineering, Michigan State University. The authors would like to thank Dr. Xi Ning for his guidance.

\section{References}

[1] B. Hamner, S. Koterba, J. Shi, R. Simmons, and S. Singh, "An autonomous mobile manipulator for assembly tasks," Autonomous Robots, vol. 28, no. 1, pp. 131-149, 2010. doi: $10.1007 /$ s10514-009-9142-y

[2] H. Yamaguchi, X. Zhaoxian, M. Aiguo, and M. Shimojo, "Human assist by a mobile manipulator with high speed tactile sensor," in IEEE International Conference on Information and Automation (ICIA), Harbin, 2010, pp. 765-770. doi: 10.1109/ICINFA.2010.5512465

[3] K. Nozaki and T. Murakami, "A motion control of two-wheels driven mobile manipulator for human-robot cooperative transportation," in The 35th Annual Conference of IEEE Industrial Electronics (IECON), Porto, Portugal, 2009, pp. 1574-1579.

doi: 10.1109/IECON.2009.5414734

[4] S. Chitta, B. Cohen, and M. Likhachev, "Planning for autonomous door opening with a mobile manipulator," in International Conference on Robotics and Automation, 2010.

Available:

http://www.willowgarage.com/sites/default/files/d oor planner.pdf

[5] T. Nishida, Y. Takemura, Y. Fuchikawa, S. Kurogi, S. Ito, M. Obata, N. Hiratsuka, H. Miyagawa, Y. Watanabe, T. Suehiro, Y. Kawamura, and F. Ohkawa,
"Development of a sensor system for outdoor service robot," in International Joint Conference SICE-ICASE, Busan, 2006, pp. 2687-2691. doi: $10.1109 /$ SICE.2006.315055

[6] S. Datta, R. Ray, and D. Banerji, "Development of autonomous mobile robot with manipulator for manufacturing environment," International Journal of Advanced Manufacturing Technology, vol. 38, no. 5-6, pp. 536-542, 2008. doi: $10.1007 / \mathrm{s} 00170-007-1024-x$

[7] A. Gasparri, S. Panzieri, F. Pascucci, and G. Ulivi, "Pose recovery for a mobile manipulator using a particle filter," in The 14th Mediterranean Conference on Control and Automation, Ancona, Italy, 2006, pp. 1-6. doi: 10.1109/MED.2006.328871

[8] A. Garcia-Cerezo, A. Mandow, J. L. Martinez, J. Gomez-de-Gabriel, J. Morales, A. Cruz, A. Reina, and J. Seron, "Development of alacrane: A mobile robotic assistance for exploration and rescue missions," in IEEE International Workshop on Safety, Security and Rescue Robotic (SSRR), Rome, Italy, 2007, pp. 1-6. doi: $10.1109 /$ SSRR.2007.4381269

[9] M. Yamashiro, X. Zhaoxian, H. Yamaguchi, M. Aiguo, and M. Shimojo, "Home service by a mobile manipulator system," in IEEE International Conference on Automation and Logistics (ICAL), Shenyang, China, 2009, pp. 295-300. doi: 10.1109/ICAL.2009.5262909

[10] N. Hai, A. Jain, C. Anderson, and C. C. Kemp, "A clickable world: Behavior selection through pointing and context for mobile manipulation," in IEEE/RSJ International Conference on Intelligent Robots and Systems (IROS), Nice, France, 2008, pp. 787-793.

doi: $10.1109 /$ IROS.2008.4651216

[11] T. Deyle, N. Hai, M. Reynolds, and C. C. Kemp, "Rf vision: RFID receive signal strength indicator (RSSI) images for sensor fusion and mobile manipulation," in IEEE/RSJ International Conference on Intelligent Robots and Systems (IROS), 2009, pp. 5553-5560. doi: $10.1109 /$ IROS.2009.5354047

[12] U. Klank, D. Pangercic, R. B. Rusu, and M. Beetz, "Real-time cad model matching for mobile manipulation and grasping," in The 9th IEEE-RAS International Conference on Humanoid Robots, 2009, pp. 290-296. doi: 10.1109/ICHR.2009.5379561

[13] I. Wulf and B. Wagner, "Fast 3D scanning methods for laser measurement systems," in International Conference on Control Systems and Computer Science (CSCS), Bucharest, Romania, 2003, pp. 312-317.

Available: http://www.rts.uni-hannover.de/images/5/5f/Wulf 03-CSCS.pdf 
[14] X. Zhiyu and W. Eryong, "Design and calibration of a fast 3D scanning LADAR," in IEEE International Conference on Mechatronics and Automation, Luoyang, Henan, 2006, pp. 211-215. doi: 10.1109/ICMA.2006.257498

[15] M. I. A. Lourakis, "A brief description of the Levenberg-Marquardt algorithm implemened," Institute of Computation Science, Foundation for research and Technology-Hellas (FORTH), Heraklion, Greece, 2005.

Available:

http://www.ics.forth.gr/ lourakis/levmar/levmar.pdf.
[16] Z. Qilong and R. Pless, "Extrinsic calibration of a camera and laser range finder (improves camera calibration)," in IEEE/RSJ International Conference on Intelligent Robots and Systems (IROS), Sendai, Japan, 2004, pp. 2301-2306.

doi: $10.1109 /$ IROS.2004.1389752

[17] P. Pérez, C. Hue, J. Vermaak, and M. Gangnet, "Color-based probabilistic tracking," in Computer vision - ECCV 2002. vol. 2350, A. Heyden, G. Sparr, M. Nielsen, and P. Johansen, Eds., Berlin; New York; Paris: Springer, 2002, pp. 661-675.

doi: $\underline{10.1007 / 3-540-47969-444}$

[18] J. Yong, X. Ning, Z. Qin, and J. Yunyi, "Target object identification and localization in mobile manipulations," in IEEE International Conference on Robotics and Biomimetics (ROBIO, Karon Beach, Phuket, 2011, pp. 144-149.

doi: $10.1109 /$ ROBIO.2011.6181276 